\title{
A Novel VLSI Design of Sign and Unsigned Irreversible and Reversible Multiplier Circuit
}

\author{
Deepmala Mishra \\ Sagar Institute of Science \& Technology, \\ Gandhinagar, Bhopal (M.P.)
}

\author{
Mohd Abdullah \\ Sagar Institute of Science \& Technology, \\ Gandhinagar, Bhopal (M.P.)
}

\begin{abstract}
Reversible rationale is all that much sought after for the future figuring advancements as they are known not low power dissemination having its applications in Low Power CMOS, Quantum Computing, Nanotechnology, and picture preparing. Adders and multipliers are fundamental building blocks in many computational units. In this paper we have presented and implemented irreversible and reversible Baugh Wooley approach using standard irreversible and reversible logic gates/cells. The problem of minimizing the number of garbage outputs is an important issue in reversible logic design. It is proved that the proposed multiplier is better and optimized, compared to its existing counterparts with respect to the number of gates, constant inputs, garbage outputs and number of transistors required.
\end{abstract}

\section{Keywords}

Irreversible Multiplier, Baugh Wooley Approach, Reversible Multiplier, Garbage Output, Quantum Cost

\section{INTRODUCTION}

In cutting edge VLSI framework power dissemination is high because of quick exchanging of inside signs. The multifaceted nature of VLSI circuits increments with every year because of pressing more rationale components into littler volumes. Consequently control dissemination has turned into the principle range of worry in VLSI plan. Reversible rationale has its rudiments from thermodynamics of data handling. As per this, customary irreversible circuits create heat because of the loss of data amid calculation. With a specific end goal to keep away from this data misfortune the ordinary circuits are displayed utilizing reversible rationale. Landauer [1961] demonstrated that the circuits outlined utilizing irreversible components scatter heat because of the loss of data bits [1]. It is demonstrated that the loss of one piece of data results in dispersal of $\mathrm{KT}^{*} \log 2$ joules of warmth vitality where $\mathrm{K}$ is the Boltzmann consistent and $\mathrm{T}$ is the temperature at which the operation is performed. Benett [1973] demonstrated that this warmth dissemination because of data misfortune can be stayed away from if the circuit is planned utilizing reversible rationale entryways [2]. An entryway is thought to be reversible if for every single info there is a novel yield task. Thus there is a coordinated mapping between the information and yield vectors. A reversible rationale entryway is a $n-$ input, n-yield gadget showing that it has same number of inputs and yields. A circuit that is constructed from reversible entryways is known as reversible rationale circuit. In this paper, we plan $5 \times 5$ piece reversible multiplier that can perform multiplier operations at the same time. Every one of the modules is reenacted in modalism SE 6.5 and integrated utilizing Xilinx ISE 14.

Utilization of reversible rationale entryways to actualize such circuits can altogether diminish the force devoured. This paper covers different viewpoints about reversible registering and reversible rationale doors. We have attempted to plan a reversible execution of eight piece math and rationale unit, ideal regarding number of doors utilized and number of waste yields delivered.

The configuration of two programmable reversible rationale entryway structures focused at ALU usage and their utilization in the acknowledgment of a proficient reversible ALU is illustrated. The ALU configuration is checked and its points of interest over the main existing ALU outline are quantitatively broke down.

Reversible rationale is all that much sought after for the future figuring advances as they are known not low power dissemination having its applications in Low Power CMOS, Quantum Computing, Nanotechnology, and Optical Computing. We have exhibited and executed reversible Wallace marked multiplier circuit in ASIC through changed Baugh-Wooley approach. Steady inputs, waste yields, equipment unpredictability, and number of transistors required are computed.

\section{BAUGH WOOLEY MULTIPLIER}

The Baugh-Wooley duplication is one of the effective techniques to handle the sign bots. This methodology has been created keeping in mind the end goal to plan normal multipliers, suited for 2's supplement numbers [2]. Let two nbit numbers, multiplier (An) and multiplicand (B), to be duplicated. An and B can be spoke to as

$$
M=-m_{n-1} 2^{n-1}+\sum_{i=0}^{n-2} m_{i} 2^{i}
$$

$$
N=-n_{n-1} 2^{n-1}+\sum_{i=0}^{n-2} n_{i} 2^{i}
$$

Where $m_{i}{ }^{\prime} s$ and $n_{i}{ }^{\prime} s$ are the bits in M and N, respectively, $m_{n-1}$ and $n_{n-1}$ are the sign bits. The product $P=M \times N$, is given by the equation:

$$
\begin{aligned}
P=M \times N= & \left(-m_{n-1} 2^{n-1}+\sum_{i=0}^{n-2} m_{i} 2^{i}\right) \\
& \times\left(-n_{n-1} 2^{n-1}+\sum_{i=0}^{n-2} n_{i} 2^{i}\right)
\end{aligned}
$$

The last item can be produced by subtracting the last two positive terms from the initial two terms [2]. Rather than doing subtraction operation, it is conceivable to acquire the 2 's supplement of the last two terms and add all terms to get 
the last item. The last two terms are bits in which every that stretch out in double weight from position up to. Then again, the last item is $2 n$ bits and reaches out in double weight from up to. At first cushion each of the last two terms in the item $P$ mathematical statement with zeros to get a 2 -bit number to have the capacity to include it with alternate terms. At that point the cushioned terms stretch out in parallel weight from up to [3].

\section{REVERSIBLE MULTIPLIER}

A reversible rationale door is a $\mathrm{n}$-information $\mathrm{n}$-yield rationale gadget with balanced mapping. This decides the yields from the inputs furthermore the inputs can be particularly recuperated from the yields. A reversible circuit ought to be planned utilizing least number of reversible rationale doors. From the perspective of reversible circuit plan, there are numerous parameters for deciding the many-sided quality and execution of circuits. The quantity of Reversible entryways (N): The quantity of reversible doors utilized as a part of circuit. The quantity of steady inputs (CI) alludes to the quantity of inputs that are to be kept up consistent at either 0 or 1 keeping in mind the end goal to integrate the given coherent capacity. The quantity of trash yields alludes to the quantity of unused yields present in a reversible rationale circuit. One can't keep away from the trash yields as these are extremely crucial to accomplish A New Approach to the Design and Quantum cost (QC) alludes to the circuit's expense as far as the expense of a primitive entryway. It is figured knowing the quantity of primitive reversible rationale entryways $(1 * 1$ or $2 * 2)$ required to understand the circuit.

To figure result of two marked numbers we have utilized adjusted Baugh-Wooley approach [8]. Both legitimate and reversible multiplier outline is partitioned into two sections: fractional item era circuit and after that multi-operand expansion circuit.

\section{Design of Logical Multiplier}

In the first place to register fractional item, we utilized 17 and 8 NAND utilizing the method given in figure 6 . After creating fractional items, next step is a multi-operand expansion. We ought to include the bits of every section given in figure 1 . To include these bits, we require FA and HA. We need to include these bits in the way that our circuit will give the best results. Figure 6 demonstrates the method for including these bits in our proposed circuit. The Wallace methodology has been utilized to build a circuit with less postpones. To minimize delay in our proposed circuit, $\mathrm{P} 9$ is registered by transforming convey yield from prior FA (FA13). The subsequent circuit for multi-operand expansion needs one 1-NOT entryway, 4HA and 16-FA.

\section{Design of Reversible Multiplier}

The operation of the $5 \times 5$ multiplier is depicted in figure 7 . It consists of 25 partial product bits of the form $X_{i} Y_{i}$. The reversible $5 \times 5$ multiplier circuit has two parts. First, the partial products are generated in parallel using Toffoli gate.

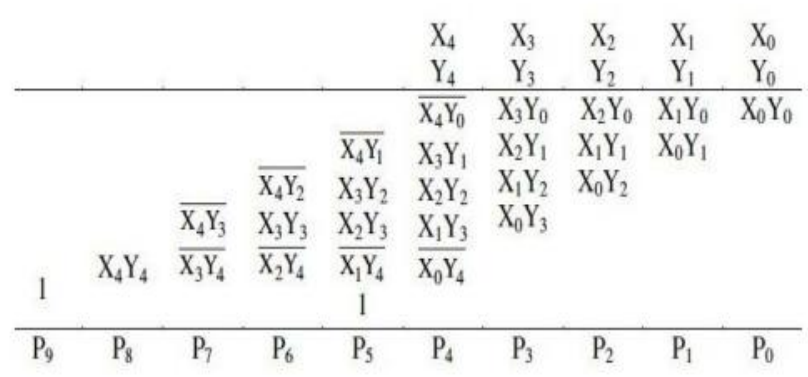

Figure 1: Baugh-Wooley 5 x 5 Signed Multiplier

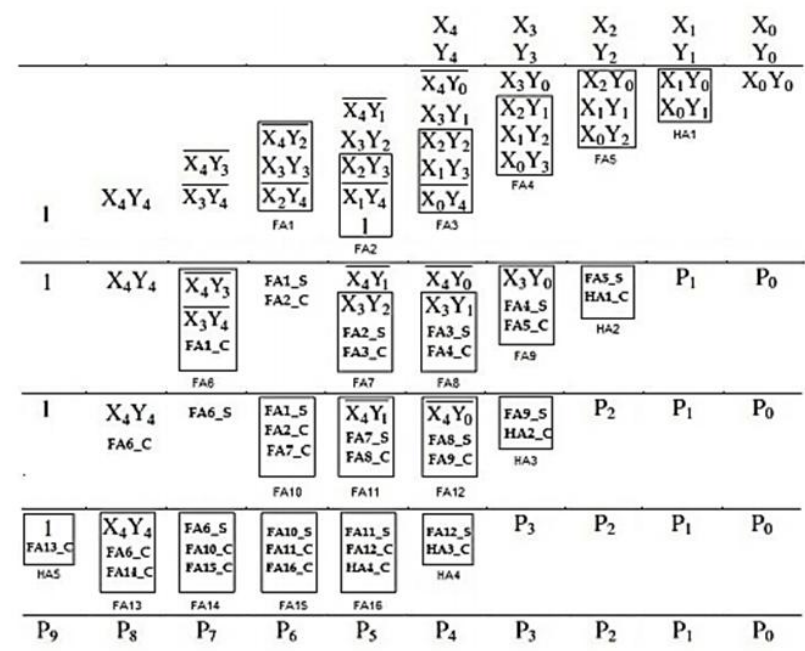

Figure 2: Multiplication by Wallace approach in the circuit

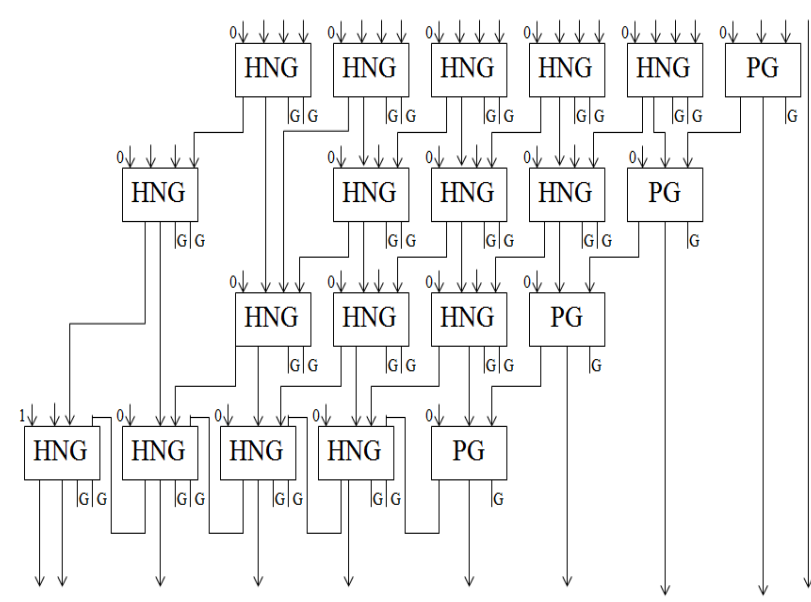

Figure 3: Multi-Operand Addition by Peres and Haghparast-Navi

We utilized 25 Toffoli entryways to make 17 ANDs and 8 NANDs as appeared in figure. The altered halfway the last low supplant by Peres entryway in light of the fact that Peres door has quantum expense of 4 as appeared if figure 3 . Next step is a multi-operand expansion. In the wake of creating incomplete items, we ought to include the bits of every section given in Hatkar A.P. et al. [6]. To include these bits, we require FA and HA. We need to include these bits in the way that our circuit to give the best results. 


\section{PROPOSED DESIGN}

The proposed reversible multiplier design into two part i.e. partial product generation by Toffoli Gates and Peres gate and multi-Operand Addition by Peres and Haghparast-Navi gates, Partial the last low replace by Peres gate because Peres gate has quantum cost of 4 as shown if figure 4 . Next step is a multi-operand addition. After generating partial products, we should add the bits of each column. To add these bits, we need FA replace by HNG gate and HA replace by Peres gate. We have to add these bits in the way that our circuit to give the best results.

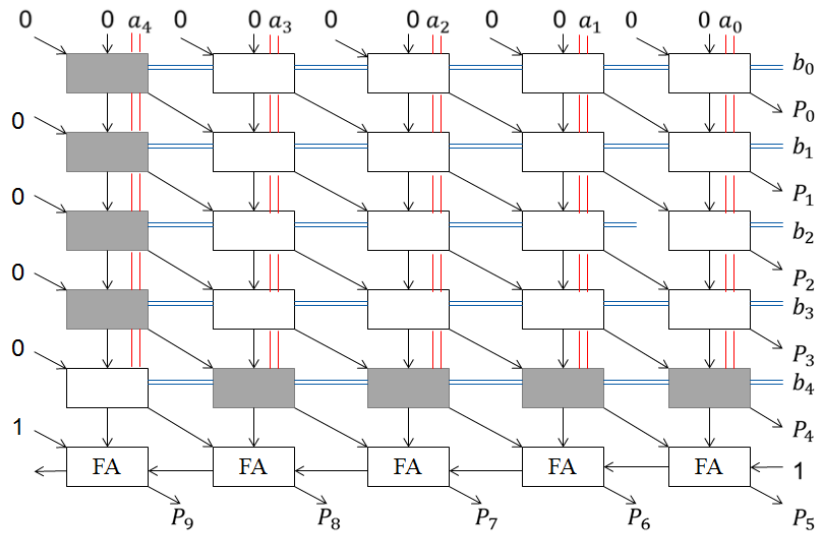

Figure 4: Proposed Design with white and gray cell
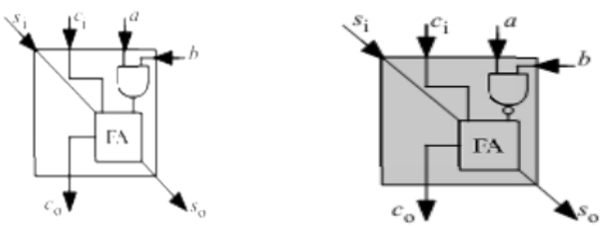

Figure 5: White and gray cell Structure

\section{SIMULATION RESULT}

The proposed irreversible and reversible multiplier circuit is more effective than the current configuration. The proposed irreversible and reversible multiplier circuit is separated two section i.e. fractional item and multi-operand expansion. The proposed halfway item is minimize 5 quantum cost in the configuration. Build the number piece of the reversible multiplier circuit so lessened the quantum cost.
Table 1: Device utilization summary Spartan-3

\begin{tabular}{|c|c|c|c|}
\hline Design & No of Slice & $\begin{array}{c}\text { No of 4 input } \\
\text { LUTs }\end{array}$ & MCPD \\
\hline $\begin{array}{c}\text { Sushma R. } \\
\text { Huddar et al. }\end{array}$ & 108 & 190 & 55.050 \\
\hline $\begin{array}{c}\text { Modified } \\
\text { Irreversible } \\
\text { Multiplier }\end{array}$ & 78 & 143 & 41.684 \\
\hline
\end{tabular}

\section{CONCLUSION}

The 1-bit, 2-bit, 4-bit, 8-bit, 16-bit and 32bit reversible ALU is designed by integrating various sub modules that includes DKG logic Gate. The performance evaluation of the various sub modules are carried out using Xilinx 14.1 ISE Simulator and it was found that the circuits designed using reversible logic showed a reduced delay and power. As a future work more arithmetic and logical function can be used.

Hence in future if reversible multiplier are used in VLSI design we can have low heat dissipation, low power and with comparatively less delay.

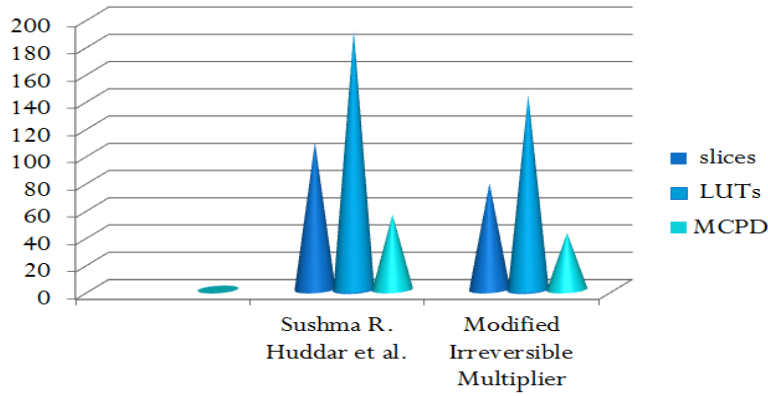

Figure 6: Bar graph of the Irreversible Multiplier

Table 2: Comparative results of reversible signed multiplier circuit

\begin{tabular}{|c|c|c|c|c|}
\hline Method & $\begin{array}{c}\text { No. of } \\
\text { slice }\end{array}$ & $\begin{array}{c}\text { No. of 4- } \\
\text { i/p LUTs }\end{array}$ & $\begin{array}{c}\text { No. of } \\
\text { I/O } \\
\text { switch }\end{array}$ & MCPD \\
\hline Proposed & 19 & 34 & 20 & 9.924 \\
\hline $\begin{array}{c}\text { Hatkar A. P } \\
\text { et al. }\end{array}$ & 21 & 37 & 20 & 10.3235 \\
\hline
\end{tabular}

In future we can have some other combination of reversible logic gates that provides more arithmetic and logical operations and hence delay can be reduced to some more extent.

Table 3: Comparative results of reversible signedmultiplier circuit

\begin{tabular}{|c|c|c|c|c|}
\hline Method & No. of Gate & $\begin{array}{c}\text { No. of garbage } \\
\text { output }\end{array}$ & $\begin{array}{c}\text { Hardware } \\
\text { Complexity }\end{array}$ & Quantum Cost \\
\hline Proposed & 45 & 46 & $25 \alpha+25 \beta$ & 232 \\
\hline $\begin{array}{c}\text { Hatkar A. P et } \\
\text { al. }\end{array}$ & 45 & 46 & $113 \alpha+61 \beta$ & 237 \\
\hline$[7]$ & 45 & 46 & $122 \alpha+61 \beta$ & 228 \\
\hline$[8]$ & 65 & 70 & $205 \alpha+105 \beta$ & - \\
\hline$[9]$ & 85 & 80 & $275 \alpha+132 \beta$ & - \\
\hline
\end{tabular}




\section{REFERENCES}

H. Thapliyal and N. Ranganathan, "Design of Efficient Reversible Binary Subtractors Based oNew Reversible Gate," Proc. of the I Computer Society Annual Symposium on VLSI, 2009.

[1] M. Morrison and N. Ranganathan, "Design of a Reversible ALU Based on Novel Programmable Reversible Logic Gate Structures," IEEE International Symposium on VLSI, 2011, pp. 126-131.

[2] Majid Haghparast, Somayyeh Jafarali Jassbi, Keivan Navi and Omid Hashemipou, "Design of a Novel Reversible Multiplier Circuit Using HNG Gate in Nanotechnology", World Applied Sciences Journal 3 (6): 974-978, 2008 ISSN 1818-4952

[3] Md. Belayet Ali, Hosna Ara Rahman and Md. Mizanur Rahman, "Design of a High Performance Reversible Multiplier", IJCSI International Journal of Computer Science Issues, Vol. 8, Issue 6, No 1, November 2011 ISSN (Online): 1694-0814.

[4] Indrayani Patle, Akansha Bhargav and Prashant Wanjari, "Implementation of Baugh-Wooley Multiplier Based on Soft-Core Processor", IOSR Journal of Engineering (IOSRJEN) e-ISSN: 2250-3021, p-ISSN: 22788719.
[5] Hatkar A. P., Hatkar A. A. and Narkhede N. P., "ASIC Design of Reversible Multiplier Circuit", 2014 International Conference on Electronic Systems, Signal Processing and Computing Technologies.

[6] H. R. Bhagyalakshmi, M. K. Venkatesha, "An Improved Design of A Multiplier Using Reversible Logic Gates," International Journal of Engineering Science and Technology, Vol. 2(8), 2010, pp.3838-3845.

[7] H. Thapliyal, M.B. Srinivas, "Novel design and reversible logic synthesis of multiplexer based full adder and multipliers", $48^{\text {th }}$ IEEE MIDWEST Symposium on Circuits and Systems (MWSCAS 2005), Cincinnati, Ohio, USA, August 7-10, 2005, pp.1593-1596.

[8] Rigui Zhou, Yang Shi, Huian Wang, Jian Cao, "Transistor realization of reversible "ZS" series gates and reversible array multiplier," Microelectronics Journal 42, 2011, pp.305-315.

[9] Oleg Golubitsky, Sean M. Falconer, and Dmitri Maslov. Synthesis of the optimal 4-bit reversible circuits. In Proceedings of the 47th Design Automation Conference, DAC '10, pages 653-656, New York, NY, USA, 2010. ACM. 